\title{
The modified ampullar approach for vestibular implant surgery: feasibility and its first application in a human with a long-term vestibular loss
}

\author{
Raymond van de Berg ${ }^{1}{ }^{*}$, Nils Guinand ${ }^{2}$, Jean-Philippe Guyot ${ }^{2}$, Herman Kingma ${ }^{1}$ and Robert J. Stokroos ${ }^{1}$ \\ ' Department of Otolaryngology and Head and Neck Surgery, Maastricht University Medical Centre, Maastricht, Netherlands \\ ${ }^{2}$ Department of ENT, University Hospital Geneva, Geneva, Switzerland
}

\section{Edited by:}

Dominik Straumann, University Hospital Zurich, Switzerland

\section{Reviewed by:}

Maurizio Versino, Pavia University, Italy

Maurizio Barbara, University La Sapienza, Italy

\section{*Correspondence:}

Raymond van de Berg, Department of Otolaryngology and Head and Neck Surgery, Maastricht University Medical Centre, Postbus 5800, 6202 AZ Maastricht, Netherlands. e-mail: raymond.vande.berg @mumc.nl

\begin{abstract}
Objective:To assess, for the first time in a human with a long-term vestibular loss, a modified approach to the ampullae and the feasibility of evoking a VOR by ampullar stimulation. Materials and methods: Peroperative stimulation of the ampullae, using the ampullar approach, was performed under full anesthesia during cochlear implantation in a 21-yearold female patient, who had experienced bilateral vestibular areflexia and sensorineural hearing loss for almost 20 years. Results: The modified ampullar approach was performed successfully with as minimally invasive surgery as possible. Ampullar stimulation evoked eye movements containing vectors congruent with the stimulated canal. As expected, the preliminary electrophysiological data were influenced by the general anesthesia, which resulted in current spread and reduced maximum amplitudes of eye movement. Nevertheless, they confirm the feasibility of ampullar stimulation. Conclusion:The modified ampullar approach provides safe access to the ampullae using as minimally invasive surgery as possible. For the first time in a human with long-term bilateral vestibular areflexia, it is shown that the VOR can be evoked by ampullar stimulation, even when there has been no vestibular function for almost 20 years. This approach should be considered in vestibular surgery, as it provides safe access to one of the most favorable stimulus locations for development of a vestibular implant.
\end{abstract}

Keywords: vestibular prosthesis, vestibular implant, neural prosthesis, bilateral vestibular areflexia, bilateral vestibulopathy, adaptation, acclimation, ampullar approach

\section{INTRODUCTION}

For more than a decade, research has been conducted into development of an invasive vestibular prosthesis (vestibular implant) (Gong and Merfeld, 2000, 2002; Lewis et al., 2002, 2010; Wall et al., 2002; Della Santina et al., 2007; Merfeld et al., 2007; Wall et al., 2007; Gong et al., 2008; Fridman et al., 2010; Davidovics et al., 2011; Guyot et al., 2011a), since non-invasive vestibular prostheses present many drawbacks in restoring vestibular function (Janssen et al., 2010). The proposed vestibular implant is analogous with the cochlear implant as it directly stimulates the neural pathways through electrical pulses (Gong and Merfeld, 2000).

Firstly, motion is detected by gyroscopes which send their signals to a processor. Secondly, the signals are processed to create an adequate stimulus with the right pulse characteristics (frequency, current, shape). Thirdly, the stimulus is delivered by electrodes to the vestibular nerve (Gong and Merfeld, 2000, 2002; Lewis et al., 2002, 2010; Wall et al., 2002; Merfeld et al., 2006, 2007; Shkel and Zeng, 2006; Della Santina et al., 2007; Fridman et al., 2010). electrically stimulated nerve branch (Gong and Merfeld, 2000, 2002; Lewis et al., 2002, 2010; Wall et al., 2002; Della Santina et al., 2007; Merfeld et al., 2007; Wall et al., 2007; Fridman et al., 2010; Davidovics et al., 2011; Guyot et al., 2011a). Moreover, the brain seems to adapt the VOR to motion modulated vestibular input and is able to increase VOR gain (maximum peak eye velocity divided by the maximum stimulus velocity), rotational axis, and symmetry during chronic stimulation (Merfeld et al., 2007; Gong et al., 2008; Fridman et al., 2010; Lewis et al., 2010). However, some hurdles have still to be overcome in relation to biomechanical and engineering issues (Wall et al., 2002). One of the main biomechanical issues is presented by the stimulus site, which is not yet properly determined. The ideal stimulus site should be a location with a well-considered compromise between three basic requirements:

1. To be close to the vestibular nerve, in order to give selective stimulation and have as little crosstalk as possible (the spread of the current to other than the targeted anatomical structures

Motion input $\longrightarrow \begin{gathered}1 . \\ \text { Sensors } \\ \text { (gyroscopes) }\end{gathered} \longrightarrow$ Processor $\stackrel{2 .}{\text { stimulus }}$ Electrodes $\longrightarrow$ Vestibular nerve

Studies have shown that it is possible to induce a nystagmus which corresponds to the plane of the canal innervated by the which leads to unintended activation of them) to the facial nerve, cochlear nerve, and other ampullary nerves (Wall et al., 
2002; Merfeld et al., 2006, 2007; Della Santina et al., 2007; Wall et al., 2007; Feigl et al., 2009; Guyot et al., 2011a).

2. To be reached with as few surgical risks as possible. Damage to the facial nerve and deafening the patient are the main risks involved in the surgical approach of some specific locations (Gacek, 1974; Parnes and McClure, 1990, 1991; Wall et al., 2007; Feigl et al., 2009; Tang et al., 2009; Dai et al., 2010).

3. To stimulate a vital part of the vestibular nerve. Studies show that, depending on pathology and elapsed time since onset of disease, different parts of the vestibular sensory system are affected to a different extent. This results in varying amounts of neurons available for the electrodes. Therefore, it is believed that different stimulus locations should be considered. Proposed locations are the ampullae, along the course of the vestibular branches, or Scarpa's ganglion (Gacek, 1974; Schuknecht, 1982; Cass et al., 1989; Leake et al., 1999; Gong and Merfeld, 2000, 2002; Tsuji et al., 2000a,b; Velazquez-Villasenor et al., 2000; Rauch et al., 2001; Lewis et al., 2002, 2010; Wall et al., 2002; Merfeld et al., 2006, 2007; Della Santina et al., 2007; Wall et al., 2007; Feigl et al., 2009; Lacour et al., 2009; McCall et al., 2009; Guyot et al., 2011a).

Previously, two types of approach have been developed for implantation of a vestibular implant:

1. An approach to the lateral ampullary nerve (LAN), superior ampullary nerve (SAN), and posterior ampullary nerve (PAN), for extralabyrinthine stimulation.

2. An approach to the ampullae of the semicircular canals (ampullar approach), for intralabyrinthine stimulation.

\section{APPROACH TO LAN/SAN/PAN}

In Geneva an approach to the PAN, LAN, and SAN has been developed and tested in humans (Wall et al., 2007; Feigl et al., 2009; Guyot et al., 2011a,b).

This surgery involves two different approaches, one for the PAN, the other one for the LAN and SAN. The PAN is reached by a transmeatal approach in which the floor of the round window niche is drilled in its most rostral part. Then, the nerve is "blue-lined" and an electrode is inserted. This technique is extracted from that described by Gacek to treat benign paroxysmal positional vertigo (Gacek, 1974; Wall et al., 2007). The LAN and SAN are reached by a transmeatal approach with removal of the head of the malleus and incus. After that, drilling is started at the spot ventral to the prominence of the lateral semicircular canal, inferior to the tegmental roof, and superior to the prominence of the facial canal. Once the nerves are reached, electrodes are inserted.

It is shown that electric stimulation from these locations induces a nystagmus which corresponds to the plane of the canal innervated by the stimulated nerve branch, and that it is possible to elicit smooth oscillatory eye movement by modulating the amplitude or frequency of the stimulation (Wall et al., 2007; Feigl et al., 2009; Guyot et al., 2011a,b).

There are a number of possible drawbacks to this type of approach. Firstly, there is a risk of sensorineural hearing loss, especially when drilling out the osseous ampulla which could result in accidental damage to the membranous labyrinth. For the approach to the PAN, the risk of sensorineural hearing loss varies from 3.6 to 38\% (Epley, 1980; Gacek and Gacek, 2002). Secondly, parts of the ossicular chain are removed and, despite ossicular chain reconstruction, a certain degree of conductive hearing loss may be caused. Thirdly, drilling is in the vicinity of the facial nerve, which carries with it the risk of damaging the nerve (Feigl et al., 2009). Fourthly, the ampullary nerves are sometimes unreachable (5-18\%; Kudo and Nomura, 1996; Feigl et al., 2009).

The main advantage, however, is the very close proximity of the electrodes to the nerves, which could allow for highly selective stimulation with little current, leading to little current spread and crosstalk (Merfeld et al., 2006; Della Santina et al., 2007; Wall et al., 2007; Feigl et al., 2009; Guyot et al., 2011a). Also, in the case of peripheral dendrites which have "died back" to Scarpa's ganglion, stimulation through this type of approach is relatively proximal to this structure, and could offer stimulation of more vital structures (Wall et al., 2002).

\section{AMPULLAR APPROACH}

In other studies, an approach to the ampullae of the semicircular canals is used (Gong and Merfeld, 2000, 2002; Lewis et al., 2002, 2010; Rubinstein and Della Santina, 2002; Merfeld et al., 2006, 2007; Gong et al., 2008; Tang et al., 2009; Fridman et al., 2010; Davidovics et al., 2011; Nie et al., 2011; Rubinstein et al., 2011). It comprises a cortical mastoidectomy, after which the semicircular canals are exposed and opened via a fenestration at the thin segment or near the junction of the thin segment and the ampulla. The electrodes are inserted and placed near the crista of the ampulla.

In animals, it is shown that it is possible to induce an electrically evoked nystagmus which corresponds to the plane of the stimulated canal. However, there are still some drawbacks that have to be investigated. For example: deafening the patient, and creating a sufficient response (Gong and Merfeld, 2000, 2002; Lewis et al., 2002, 2010; Rubinstein and Della Santina, 2002; Merfeld et al., 2006, 2007; Gong et al., 2008; Tang et al., 2009; Dai et al., 2010; Fridman et al., 2010). When the canal is fenestrated, perilymph oozes out of the canal. Depending on the case, sensorineural hearing loss could occur, which is not always reversible (Parnes and McClure, 1990, 1991; Tang et al., 2009; Dai et al., 2010). Regarding a sufficient response, as stated earlier, a vital part of the vestibular nerve should be stimulated. When peripheral dendrites have "died back" to Scarpa's ganglion (Wall et al., 2002), a location near the ampulla could be too far from a vital structure to create a sufficient response.

The main advantages of this type of approach are that the facial nerve remains relatively safe from damage and that the middle ear structures are preserved (Wall et al., 2007; Feigl et al., 2009; Guyot et al., 2011a).

The ampullar approach appears to be relatively safe, with few drawbacks, and easier to use than the LAN/SAN/PAN-approach. However, the drawbacks and possible risks of the ampullar approach, namely more current spread, sensorineural hearing loss (Tang et al., 2009; Dai et al., 2010), and damage to the facial nerve and ossicular chain, have not yet been extensively investigated in humans. A few studies have shown that, using the appropriate surgical technique and equipment, there is a risk of hearing loss, but that this is neither severe nor permanent (Parnes and McClure, 1990, 1991; Gacek and Gacek, 2002; Tang et al., 2009; Dai et al., 2010; Rubinstein et al., 2011). Also unknown is whether 
it is possible to stimulate a vital part of the nerve, when there has not been any vestibular function for many years. Therefore, the goal of this study was to evaluate for the first time, in a human with a long-term vestibular loss, the ampullar approach, and the feasibility of evoking a VOR by ampullar stimulation. This was achieved by:

1. Performing the ampullar approach in a human, and specifying/modifying the technique in order to minimize surgical drilling and damage to the otic capsule;

2. Showing and discussing the preliminary results of ampullar stimulation under general anesthesia.

\section{MATERIALS AND METHODS SURGICAL PROCEDURE: THE AMPULLAR APPROACH}

The ampullar approach has previously been performed by using a cortical mastoidectomy, exposition of the semicircular canals, extensive bluelining of the semicircular canals, and fenestrating them at the thin segment or adjacent to the ampullary ends (Gong and Merfeld, 2000, 2002; Lewis et al., 2002, 2010; Rubinstein and Della Santina, 2002; Merfeld et al., 2006, 2007; Gong et al., 2008; Tang et al., 2009; Fridman et al., 2010; Davidovics et al., 2011; Nie et al., 2011; Rubinstein et al., 2011). In this trial, the approach was specified more clearly, since the use of fixed anatomical markers as a reference prevents the surgeon from drilling unnecessarily and minimizes damage to the otic capsule. Drilling was performed in a direct angle to the ampullae, without bluelining relatively large portions of the canals, resulting in as minimally invasive surgery as possible and, therefore, minimizing the chance of accidentally entering the canals.

Intra-operative facial nerve monitoring was used.

\section{Approach to the ampullae of the superior and lateral canal}

When the superior and lateral semicircular canals fuse at the vestibule, they make a "V-shape." Therefore, after the mastoidectomy and posterior tympanotomy, minimally invasive bluelining of the anterior ends of the canals (adjacent to each ampullary end) was obtained by drilling cranially at the dome of the lateral canal and following it, until the " $V$ " appeared. A small diamond burr $(2 \mathrm{~mm})$ was used. As the anterior end of the superior canal was located by following the lateral canal, no more drilling of the superior canal was necessary. Eventually, approximately only $3 \mathrm{~mm}$ of the anterior ends of both canals were blue-lined. After that, a small fenestration adjacent to each of the ampullary ends was made. In this case, a manually positioned temporary electrode was inserted (see below). Permanent electrodes would be inserted in the case of a vestibular implant (Figures 1 and 2 ).

\section{Approach to the posterior ampulla}

The ampulla of the posterior canal is located nearby the oval window and stapes. Therefore, it is located medial to the facial nerve, at an imaginary almost horizontal line through the stapes footplate, between the sigmoid sinus and the facial nerve (Figure 1, line $\mathrm{X})$. Using the stapes as a reference, minimally invasive surgery was obtained by directly undermining the facial nerve below the lateral semicircular canal and drilling a hole in the direction of the stapes. After bluelining the anterior end of the canal, a small fenestration
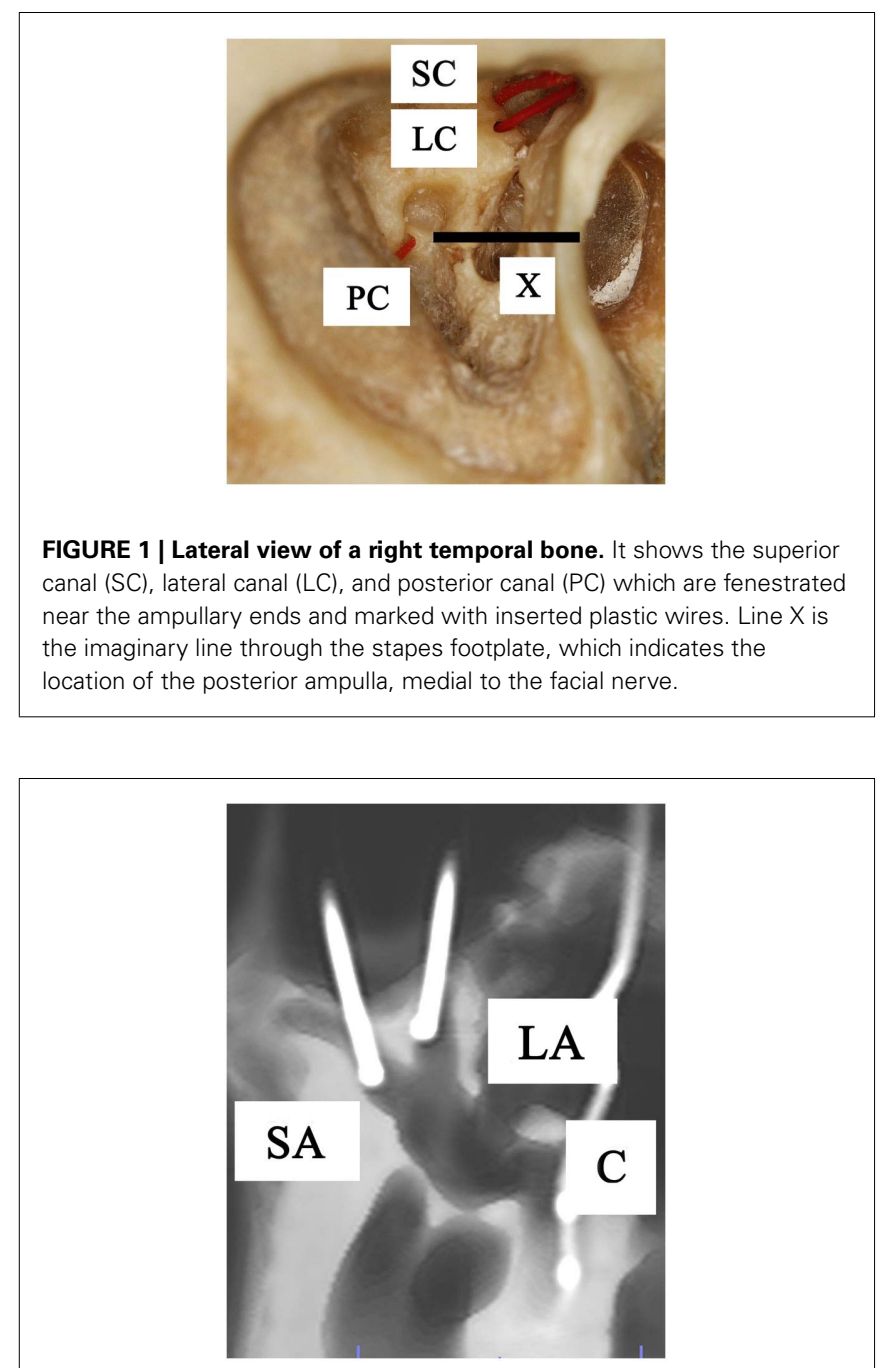

FIGURE 2 | Anterolateral view of a right temporal bone using cone beam CT-scan. It shows the fenestration and insertion of the cochleovestibular electrodes in the superior ampulla (SA), lateral ampulla (LA), and cochlea (C).

adjacent to the ampulla was made. Again, temporary electrodes were then inserted (Figure 3). However, it would also be possible to insert permanent electrodes, according to the requirements of the case.

\section{HUMAN SUBJECT}

The subject of this experiment was a 21-year-old woman undergoing surgery for cochlear implantation, performed by the lastnamed author in Maastricht University Medical Centre. Due to meningitis in her childhood, she became bilaterally deaf with bilateral vestibular areflexia. She reported no vestibular complaints.

Three inclusion criteria were fulfilled:

1. mean peak slow phase velocity of $\leq 5^{\circ} / \mathrm{s}$ in bilateral bithermal caloric irrigations; 


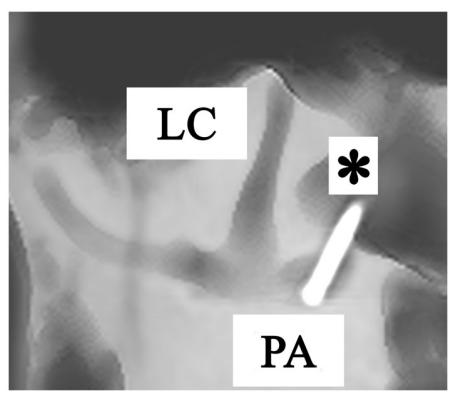

FIGURE 3 | Anterolateral view of a right temporal bone using cone beam CT-scan. It shows the fenestration and insertion of the electrode (*) in the posterior ampulla (PA). ( $\mathrm{LC}=$ lateral canal).

2. pathological Head-Impulse-Test (HIT) for horizontal and vertical canals;

3. low or no gain on rotatory chair tests.

Electro-nystagmography (ENG) was used for vestibular testing. Bilateral bithermal $\left(30^{\circ}\right.$ and $\left.44^{\circ}\right)$ caloric irrigations were performed by experienced technicians in standard conditions. Rotatory chair tests consisted of horizontal and vertical torsion swing $(0.11 \mathrm{~Hz}, \omega \max =100 \%$ s $)$ and bilateral velocity steps $\left(\omega=250^{\circ} / \mathrm{s}\right)$. Manual HITs were recorded with a high speed camera [CASIO Exilim, Pro EX-F1, 12× optical zoom, high speed camera, 300 frames per second (fps)] in the three semicircular canal planes. The presence of correction saccades was considered as pathological. She showed no response to bilateral vestibular galvanic stimulation. These vestibular tests were only performed pre-operatively.

Informed consent, and approval from the Medical Ethical Committee, in accordance with the Helsinki Declaration, were obtained (protocol name and number: "Electric stimulation of the ampullary nerves in patients with bilateral vestibular loss" NL31405.068.10; World Medical Association General Assembly, 2000). The study was registered at the Dutch Trial Register: www.trialregister.nl.

\section{EXPERIMENTAL TESTING}

\section{Stimulation equipment, paradigm}

To check the locations of the fenestrations, and to show the feasibility of evoking a VOR by stimulating the ampullary nerves via the ampullae, a monopolar electrode was used to electrically stimulate the ampullae. It was manually positioned on the cristae of the ampullae for intralabyrinthine stimulation. The return electrode was placed on the patient's back. When measurements were completed, the canals were closed with bone wax and Tissucol ${ }^{\circledR}$ (Baxter, aprotinin, trombin).

Matlab $\odot$ software was used to drive a real-time processor (RP2.1 Real-Time Processor, Tucker-Davis Technologies@) which was connected to a galvanic stimulator (Maastricht Instruments bv() where the stimulus was converted to a current. A monopolar stimulator (Standard Prass Flush-Tip Stimulator Probe, Medtronic (C) of $500 \mu \mathrm{m}$ was used to deliver the current to the nerves.

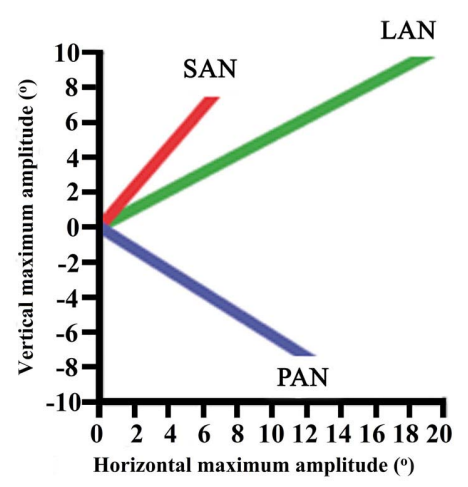

FIGURE 4 | Maximum vertical and horizontal amplitudes of the eye during ampullar nerve stimulation of each canal.

Biphasic pulses of $200 \mu$ s phase duration with a repetition rate of $200 \mathrm{~Hz}$ were used. Amperage was modulated step by step from 0 to a maximum of $1 \mathrm{~mA}$. Pulse train duration was $10 \mathrm{~s}$ with on/off periods of $0.5 \mathrm{~s}$.

\section{Eye movements, recording, and analysis}

The horizontal, vertical, and torsional eye movements were recorded using video oculography (Clinical Video Eye Tracker, Maastricht Instruments bv()) at 50 samples/s. Recordings were analyzed off-line with algorithms written in Matlab $\odot$ software. Horizontal, vertical, and torsional components were estimated at suprathreshold stimulation parameters.

\section{Anesthetics}

The whole procedure was performed under general anesthesia. Propofol $6 \mathrm{mg} / \mathrm{kg} / \mathrm{h}$ and Remifentanil $0.35 \mathrm{mcg} / \mathrm{kg} / \mathrm{min}$ were administered during the surgical approach. When electric stimulation and measurements commenced, Propofol was stopped, and Remifentanil was continued at the same dosage. After electric stimulation, the cochlear implantation procedure was resumed with Propofol only $(8 \mathrm{mg} / \mathrm{kg} / \mathrm{h})$.

\section{RESULTS}

The modified ampullar approach was performed successfully: the ampullae from the anterior, lateral, and posterior canal were reached, with minimal bluelining of the anterior ends of the canals. No damage to the facial nerve, ossicular chain, or inner ear structures was observed.

Stimulation with $700 \mu \mathrm{A}$ elicited tonic eye deviation in all three ampullae, confirming the positioning of the electrode and feasibility of ampullar stimulation by this approach.

Maximum vertical and horizontal amplitudes of the eye during ampullar nerve stimulation of the canals ranged from $6.6^{\circ}$ to $19^{\circ}$. These results are presented for each canal in Figure 4.

Stimulation of the LAN showed a horizontal component which was away from the stimulation side. The vertical component was upward. For the stimulation of the SAN, the vertical component was upward and the horizontal component was away from the stimulation side. Maximum amplitude of torsion was $17^{\circ}$. Stimulation of the PAN elicited tonic eye deviation with a downward 


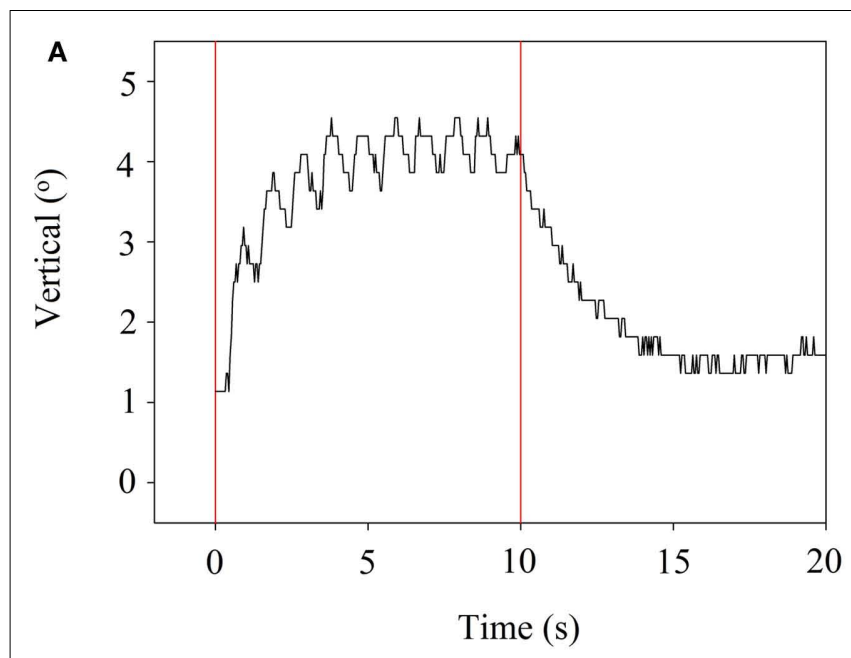

B

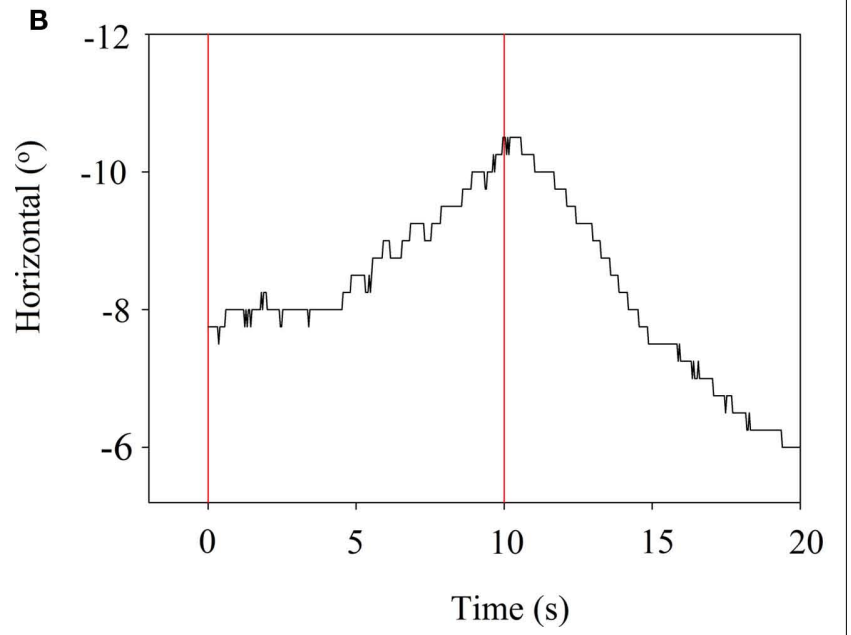

FIGURE 5 | (A) VOR vertical component during suprathreshold stimulation of the SAN. Vertical bars indicate the start and end of stimulation. (B) VOR horizontal component during suprathreshold stimulation of the SAN. Vertical bars indicate the start and end of stimulation.

vertical component and a horizontal component away from the stimulation side. When the current was increased to obtain maximum amplitudes, facial twitching was observed in most of the cases.

When stimulation was stopped, the eye returned back to its starting position. Examples are presented in Figures 5A,B, which refer to stimulation of the SAN. The horizontal component is delayed ( $5 \mathrm{~s}$ ) compared to the vertical component.

Regarding the anesthetics, it was clearly observed that after the Propofol was stopped, reactions became more profound once the Propofol was cleared from the body.

During post-operative follow-up, the patient did not suffer from any change in vestibular symptoms. Facial nerve function was preserved.

\section{DISCUSSION}

This study shows, for the first time in a human with a long-term vestibular loss, the modified ampullar approach with minimally invasive surgery and the feasibility of ampullar stimulation. There is an important difference between this human subject and subjects in previous research: previous subjects still had some residual function, or bilateral vestibulopathy was induced by canal plugging or ototoxic medication prior to implantation (Gong and Merfeld, 2002; Lewis et al., 2002, 2010; Wall et al., 2002; Merfeld et al., 2006, 2007; Della Santina et al., 2007; Gong et al., 2008; Tang et al., 2009; Dai et al., 2010, 2011; Davidovics et al., 2011; Rubinstein et al., 2011). Our human subject had exhibited no vestibular function for almost 20 years. Therefore, it was very important to show whether stimulation in this subject would be feasible. This study shows that a VOR can be evoked by electrically stimulating the ampullae under general anesthesia, in a human with a long-term vestibular loss, and in whom galvanic stimulation did not elicit any response during vestibular testing pre-operatively. This suggests that stimulation of the ampullae is probably close enough to vital parts of the vestibular nerve to elicit a response in the majority of cases. "Dying back" of the nerves could be less of an issue than was previously assumed. However, it could influence the stimulation range (change of stimulation thresholds) and inter-individual differences in responses to the vestibular implant.

The modified ampullar approach seems to be a safe approach. Firstly, the facial nerve remains relatively safe from damage when intra-operative facial nerve monitoring is employed. Secondly, middle and inner ear structures are preserved. Thirdly, surgery of the vestibular system is already well known by neurootologic surgeons compared to the extralabyrinthine approach to the LAN/SAN/PAN. Fourthly, accidental damage to the canals is reduced, by modifying the ampullar approach into a procedure with minimally invasive surgical drilling, using the "V-shape" of the superior and lateral canal, together with the imaginary horizontal line through the stapes footplate as a reference. This facilitates safe bluelining of only the anterior ends of the canals, adjacent to the ampullary ends. Further advantages of the ampullar approach are that multiple electrodes can be inserted into the ampulla, and that fixating them will be less challenging than with the extralabyrinthine approach. For example, if the array of electrodes shifts accidentally during surgery, another electrode with the best response could be selected for stimulation. In addition, using more electrodes could also facilitate current-steering and precompensation (correcting the misalignment of the VOR-axis by vector summation; Fridman et al., 2010).

One potential disadvantage of intralabyrinthine ampullar stimulation is the general belief of deafening the patient. However, more studies show that, with appropriate surgical technique and equipment, opening the canals carries a risk of hearing loss that is neither severe nor permanent (Parnes and McClure, 1990, 1991; Gacek and Gacek, 2002; Tang et al., 2009; Dai et al., 2010; Rubinstein et al., 2011). In the future, intralabyrinthine stimulation could thus become an option in hearing patients, after more thorough investigations in humans. Therefore, the ampullar approach should be considered in vestibular implant surgery since it carries low risks. It opens up new perspectives on the development of a $3 \mathrm{D}$ vestibular implant with selective intralabyrinthine stimulation of the vestibular nerve.

It should be noted that the ampullar approach does not rule out the extralabyrinthine approach to the LAN/SAN/PAN. They 
could be used as complementary procedures (a combination of both approaches in one patient) or as alternatives (the appropriate technique is selected for each patient), depending on the results of future research.

These preliminary results show that ampullar stimulation is possible in a person with a long-term vestibular loss. Stimulation leads to a tonic eye deviation with mixed components and variable maximum amplitudes. Although components are mixed, stimulation always evokes a vector which is congruent with the canal: a horizontal component during LAN-stimulation, an upward component during SAN-stimulation, and a downward component during PAN-stimulation. This component is not always the predominant vector. However, in Figure 4 it is shown that stimulation of each ampulla has its own vector. Studies show that this is more important than having the right vector, since cross-axis adaptation can occur. This is the phenomenon that eye responses which are aligned with the axis of head motion, can be evoked by stimulating a canal which is orthogonal to the axis of motion (Lewis et al., 2002, 2010; Della Santina et al., 2007; Dai et al., 2011). Research in chinchillas showed that there was a significant improvement of the VOR-axis after 1 week of multichannel prosthetic stimulation, indicating that the central nervous system rapidly adapts to prosthetic stimulation (Dai et al., 2011).

Crosstalk is an important factor. Figure 5B shows a delayed horizontal component (after $5 \mathrm{~s}$ ) during SAN-stimulation, suggesting current spread resulting in delayed recruitment of the LAN. In addition, Figure 4 shows mixed components during ampullar stimulation as a result of significant crosstalk between the ampullary and/or otolith nerves. However, it is difficult to interpret the crosstalk between nerves. For example, the horizontal component of SAN-stimulation could be the result of simultaneous LAN-stimulation, utricular stimulation, or both. Factors which could play a role in the misalignment are both the pulse duration and the current. In this experiment, biphasic pulses of $200 \mu \mathrm{s}$ phase duration and $10 \mathrm{~s}$ pulse train duration were used. Animal research has shown that misalignment is reduced by using a lower pulse duration and current (Davidovics et al., 2011). In future research, pulse phase and train duration could be lowered, especially as a pulse train duration of $10 \mathrm{~s}$ does not mimic physiological conditions. As presented in Figure 5B, the horizontal component is not visible until $4-5 \mathrm{~s}$ after SAN-stimulation. Normal head motions do not last for $5 \mathrm{~s}$. This could suggest that during physiological conditions, in which head motions generally have a shorter duration, recruitment of the other ampullary nerves is less likely as a shorter time does not allow the current spread to reach the other nerves. Regarding current, a higher current was necessary in order to cope with the effects of general anesthesia. This resulted in current spread to the other ampullary and/or otolith nerves as well as to the facial nerve. Once a prosthesis is

\section{REFERENCES}

Cass, S. P., Davidson, P., and Goshgarian, H. (1989). Survival of the vestibular nerve after labyrinthectomy in the cat. Otolaryngol. Head Neck Surg. 101, 459-465.

Dai, C., Fridman, G. Y., Chiang, B., Davidovics, N. S., Melvin, T. A.,

implanted, it is believed that a lower current could be maintained during daily use, resulting in less current spread. If misalignment of a vestibular prosthesis should remain after adjusting for the factors mentioned above, precompensation could also be used since it has been shown to significantly restore the VOR-axis (Fridman et al., 2010).

We were able to evoke a VOR under full anesthesia by replacing Propofol by Remifentanil. This is a useful finding since, at least in our clinic, both patients and ethical committees are more likely to cooperate when surgical procedures and investigations are performed under general anesthesia. Although anesthesia will influence the reactions to electrical stimuli (Poon and Irwin, 2009) (e.g., changing thresholds and stimulation range, lowering maximum amplitudes of eye movements), this anesthetic protocol can be used in future investigations. It could facilitate vestibular implant surgery when intra-operative ampullar stimulation is necessary to investigate electrode position or when electrically evoked compound action potentials (ECAPs) are not obtained during surgery (Nie et al., 2011). The feasibility of applying ampullar stimulation in cases of vestibular nerve degeneration could also be assessed. However, it can only be used for qualifying, and not for quantifying, electrophysiological reactions. For example, under normal conditions, maximum amplitudes of $6.6^{\circ}-19^{\circ}$ would be suboptimal for the use of a vestibular implant. When Propofol was stopped, reactions became more profound once the Propofol was cleared from the body. The effect of Remifentanil remained. Therefore, we can only state that stimulation is feasible; realistic maximum amplitudes cannot be given. However, the maximum amplitudes that were obtained under general anesthesia seem very promising.

\section{CONCLUSION}

The modified ampullar approach provides safe access to the ampullae using as minimally invasive surgery as possible. For the first time in a human with long-term bilateral vestibular areflexia, it has been shown that the VOR can be evoked by ampullar stimulation, even when there has been no vestibular function for almost 20 years. This approach should be considered in vestibular surgery, since it provides safe access to one of the most favorable stimulus locations for development of a vestibular implant.

\section{ACKNOWLEDGMENTS}

The authors wish to acknowledge Dr. A. E. W. Hamaekers and Dr. J. Jansen for their contributions to the anesthetic protocol, S. M. H. Jansen for his contributions to the dataanalysis, and G. S. Townend for her textual criticism. Also, they wish to thank the reviewers for their valuable comments and suggestions.

Dai, C., Fridman, G. Y., and Della Santina, C. C. (2010). Effects of vestibular prosthesis electrode implantation and stimulation on hearing in rhesus monkeys. Hear. Res. 277, 204-210.

Davidovics, N. S., Fridman, G. Y., Chiang, B., and Della Santina,
C. C. (2011). Effects of biphasic current pulse frequency, amplitude, duration, and interphase gap on eye movement responses to prosthetic electrical stimulation of the vestibular nerve. IEEE Trans. Neural Syst. Rehabil. Eng. 19, 84-94. 
Della Santina, C. C., Migliaccio, A. A., and Patel, A. H. (2007). A multichannel semicircular canal neural prosthesis using electrical stimulation to restore 3-D vestibular sensation. IEEE Trans. Biomed. Eng. 54, 1016-1030.

Epley, J. M. (1980). Singular neurectomy: hypotympanotomy approach. Otolaryngol. Head Neck Surg. 88, 304-309.

Feigl, G. C., Fasel, J. H., Anderhuber, F., Ulz, H., Rienmüller, R., Guyot, J. P., and Kos, I. M. (2009). Superior vestibular neurectomy: a novel transmeatal approach for a denervation of the superior and lateral semicircular canals. Otol. Neurotol. 30, 586-591.

Fridman, G. Y., Davidovics, N. S., Dai, C., Migliaccio, A. A., and Della Santina, C. C. (2010). Vestibulo-ocular reflex responses to a multichannel vestibular prosthesis incorporating a 3D coordinate transformation for correction of misalignment. J. Assoc. Res. Otolaryngol. 11, 367-381.

Gacek, R. R. (1974). Transection of the posterior ampullary nerve for the relief of benign paroxysmal positional vertigo. Ann. Otol. Rhinol. Laryngol. 83, 596-605.

Gacek, R. R., and Gacek, M. R. (2002). Results of singular neurectomy in the posterior ampullary recess. ORL J. Otorhinolaryngol. Relat. Spec. 64, 397-402.

Gong, W., Haburcakova, C., and Merfeld, D. M. (2008). Vestibulo-ocular responses evoked via bilateral electrical stimulation of the lateral semicircular canals. IEEE Trans. Biomed. Eng. 55, 2608-2619.

Gong, W., and Merfeld, D. M. (2000). Prototype neural semicircular canal prosthesis using patterned electrical stimulation. Ann. Biomed. Eng. 28, 572-581.

Gong, W., and Merfeld, D. M. (2002). System design and performance of a unilateral horizontal semicircular canal prosthesis. IEEE Trans. Biomed. Eng. 49, 175-181.

Guyot, J. P., Sigrist, A., Pelizzone, M., Feigl, G. C., and Kos, M. I. (2011a). Eye movements in response to electrical stimulation of the lateral and superior ampullary nerves. Ann. Otol. Rhinol. Laryngol. 120, 81-87.
Guyot, J. P., Sigrist, A., Pelizzone, M., and Kos, M. I. (2011b). Adaptation to steady-state electrical stimulation of the vestibular system in humans. Ann. Otol. Rhinol. Laryngol. 120, 143-149.

Janssen, M., Stokroos, R., Aarts, J., van Lummel, R., and Kingma, H. (2010). Salient and placebo vibrotactile feedback are equally effective in reducing sway in bilateral vestibular loss patients. Gait Posture 31, 213-217.

Kudo, Y., and Nomura, Y. (1996). The vestibular nerve: its course to the anterior and lateral ampullae. $O R L$ J. Otorhinolaryngol. Relat. Spec. 58, 208-212.

Lacour, M., Dutheil, S., Tighilet, B. Lopez, C., and Borel, L. (2009). Tell me your vestibular deficit, and i'll tell you how you'll compensate. Ann. N. Y. Acad. Sci. 1164, 268-278.

Leake, P. A., Hradek, G. T., and Snyder, R. L. (1999). Chronic electrical stimulation by a cochlear implant promotes survival of spiral ganglion neurons after neonatal deafness. $J$. Comp. Neurol. 412, 543-562.

Lewis, R. F., Gong, W., Ramsey, M., Minor, L., Boyle, R., and Merfeld, D. M. (2002). Vestibular adaptation studied with a prosthetic semicircular canal. J. Vestib. Res. 12, 87-94.

Lewis, R. F., Haburcakova, C., Gong, W., Makary, C., and Merfeld, D. M. (2010). Vestibuloocular reflex adaptation investigated with chronic motion-modulated electrical stimulation of semicircular canal afferents. J. Neurophysiol. 103, 1066-1079.

McCall, A. A., Ishiyama, G. P., Lopez, I. A., Bhuta, S., Vetter, S., and Ishiyama, A. (2009). Histopathological and ultrastructural analysis of vestibular end organs in Meniere's disease reveals basement membrane pathology. BMC Ear Nose Throat Disord. 9, 4. doi:10.1186/1472-6815-9-4.

Merfeld, D. M., Gong, W., Morrissey, J., Saginaw, M., Haburcakova, C., and Lewis, R. F. (2006). Acclimation to chronic constant-rate peripheral stimulation provided by a vestibular prosthesis. IEEE Trans. Biomed. Eng. 53, 2362-2372.

Merfeld, D. M., Haburcakova, C., Gong, W., and Lewis, R. F. (2007). Chronic vestibulo-ocular reflexes evoked by a vestibular prosthesis. IEEE Trans. Biomed. Eng. 54, 1005-1015.
Nie, K., Bierer, S. M., Ling, L., Oxford, T., Rubinstein, J. T., and Phillips, J. O. (2011). Characterization of the electrically evoked compound action potential of the vestibular nerve. Otol. Neurotol. 32, 88-97.

Parnes, L. S., and McClure, J. A. (1990). Posterior semicircular canal occlusion for intractable benign paroxysmal positional vertigo. Ann Otol. Rhinol. Laryngol. 99, 330-334.

Parnes, L. S., and McClure, J. A. (1991) Posterior semicircular canal occlusion in the normal hearing ear. Otolaryngol. Head Neck Surg. 104 52-57.

Poon, C. C., and Irwin, M. G. (2009). Anaesthesia for deep brain stimulation and in patients with implanted neurostimulator devices. Br. J. Anaesth. 103, 152-165.

Rauch, S. D., Velazquez-Villasenor, L. Dimitri, P. S., and Merchant, S. N. (2001). Decreasing hair cell counts in aging humans. Ann. N. Y. Acad. Sci. 942, 220-227.

Rubinstein, J. T., and Della Santina, C. C. (2002). Development of a biophysical model for vestibular prosthesis research. J. Vestib. Res. 12, 69-76.

Rubinstein, J. T., Phillips, J. O. Nie, K., Ling, L., Bierer, S., and Golub, J. (2011). Feasibility Studies of the UW/Nucleus Vestibular Implant for Meniere's Disease: First Human Data Collegium Oto-RhinoLaryngologicum Amicitae Sacrum. Brugge.

Schuknecht, H. F. (1982). Behavior of the vestibular nerve following labyrinthectomy. Ann. Otol. Rhinol. Laryngol. Suppl. 97, 16-32.

Shkel, A. M., and Zeng, F. G. (2006). An electronic prosthesis mimicking the dynamic vestibular function. Audiol. Neurootol. 11, 113-122.

Tang, S., Melvin, T. A., and Della Santina, C. C. (2009). Effects of semicircular canal electrode implantation on hearing in chinchillas. Acto Otolaryngol. 129, 481-486.

Tsuji, K., Velazquez-Villasenor, L. Rauch, S. D., Glynn, R. J., Wall, C. III, and Merchant, S. N. (2000a). Temporal bone studies of the human peripheral vestibular system. Aminoglycoside ototoxicity. Ann. Otol. Rhinol. Laryngol. Suppl. 181, 20-25.

Tsuji, K., Velazquez-Villasenor, L., Rauch, S. D., Glynn, R. J., Wall, C.
III, and Merchant, S. N. (2000b). Temporal bone studies of the human peripheral vestibular system. Meniere's disease. Ann. Otol. Rhinol. Laryngol. Suppl. 181, 26-31.

Velazquez-Villasenor, L., Merchant, S. N., Tsuji, K., Glynn, R. J., Wall, C. III and Rauch, S. D. (2000). Temporal bone studies of the human peripheral vestibular system. Normative Scarpa's ganglion cell data. Ann. Otol. Rhinol. Laryngol. Suppl. 181, 14-19.

Wall, C. III, Kos, M. I., and Guyot, J. P. (2007). Eye movements in response to electric stimulation of the human posterior ampullary nerve. Ann. Otol. Rhinol. Laryngol. 116, 369-374.

Wall, C. III, Merfeld, D. M., Rauch, S. D., and Black, F. O. (2002). Vestibular prostheses: the engineering and biomedical issues. J. Vestib. Res. 12, 95-113.

World Medical Association General Assembly. (2000). World medical association declaration of Helsinki: ethical principles for medical research involving human subjects. JAMA 284, 3043-3045.

Conflict of Interest Statement: The authors declare that the research was conducted in the absence of any commercial or financial relationships that could be construed as a potential conflict of interest.

Received: 04 December 2011; paper pending published: 06 January 2012; accepted: 30 January 2012; published online: 20 February 2012.

Citation: van de Berg R, Guinand $N$, Guyot J-P, Kingma $H$ and Stokroos RJ (2012) The modified ampullar approach for vestibular implant surgery: feasibility and its first application in a human with a long-term vestibular loss. Front. Neur. 3:18. doi: 10.3389/fneur.2012.00018

This article was submitted to Frontiers in Neuro-otology, a specialty of Frontiers in Neurology.

Copyright (C) 2012 van de Berg, Guinand, Guyot, Kingma and Stokroos. This is an open-access article distributed under the terms of the Creative Commons Attribution Non Commercial License, which permits non-commercial use, distribution, and reproduction in other forums, provided the original authors and source are credited. 